\title{
Contemporary liver surgery
}

\author{
Stefan Stättner
}

(C) Springer-Verlag GmbH Austria, part of Springer Nature 2018

Dear valued reader,

Liver surgery has dramatically improved in the past 30 years. This is mainly reflected by dramatically reduced rates of perioperative mortality. Delivering safe surgery opens new fields of surgical indications in cancer surgery and a complete paradigm shift to the future liver remnant approach-"what counts is what remains". Hence, dedicated hepatobiliary surgeons have increased their input in multidisciplinary team discussions to gradually expand indications for surgery. Although randomized prospective data are generally still lacking, numerous series on the effect of hepatectomy on patients' outcome have been published; therefore, performing trials randomizing patients into the non-surgical group is problematic, as patients might refuse to take part in such a study. With more available systemic and local treatment options and a better molecular-based understanding of the diseases, surgeons have to learn and incorporate this knowledge when assessing whether a resection is biologically feasible or not. Liver surgery in 2018 is one piece of a puzzle of various treatment strategies which needs careful implementation in this complex multistep treatment approach.

New strategies like ALPPS (associating liver partition and portal vein ligation for staged hepatectomy) have led to numerous scientific discussions and have split our hepatobiliary community in groups of pro and con, mainly because of the high mortality at the beginning of its era, which now seems to have decreased a few years after the first description, at least

PD Dr. S. Stättner, FRCS, FEBS ( $\triangle)$

Department of Visceral, Transplantation and

Thoracic Surgery, Medical University of Innsbruck,

Anichstraße 35, 6020 Innsbruck, Austria

stefan.staettner@i-med.ac.at in specialised centres. Nevertheless, it has opened a new field of a potential salvage therapy and gives new insights into the dynamics of liver regeneration.

This special edition on contemporary liver surgery is dedicated to general surgeons, visceral and hepatobiliary surgeons as well as medical oncologists and general practitioners who are facing the potential surgical candidate with liver disease. Gasteiger et al. describe important issues on the background of liver disease as well as the importance of perioperative management and anaesthetic care for good outcomes after complex liver resections. This team has major experience in adult and paediatric liver transplantation as well. A well-sorted selection of surgical candidates improves outcomes, as does postoperative enhanced recovery after surgery (ERAS). Quinn and Jones et al. from the renowned group in Liverpool/UK, who are pioneers in ERAS and liver surgery, review current literature and share their expertise for colorectal cancer liver metastases management and perihilar cholangiocarcinoma patients in their manuscripts.

Surgical treatment is usually divided into benign and malignant diseases, amongst the latter, primaries are separated from metastatic lesions. These important topics are extensively covered by two large liver centres from Austria (Braunwarth et al., Fodor et al. and Schwarz et al.).

Interventional oncology is clearly on the rise and increasingly used in many centres. Due to its minimally invasive approach, it is an important treatment option and an especially attractive modality from the patients' point of view. Putzer et al. have reviewed the current data and present their extensive experience with stereotactic radiofrequency ablation (sRFA).

The journey ends with outlooks into the future. Regeneration is a fantastic peculiarity of the liver which is still only poorly understood. Pereyra and Starlinger accepted to take on this interesting chapter and pro- 


\section{editorial}

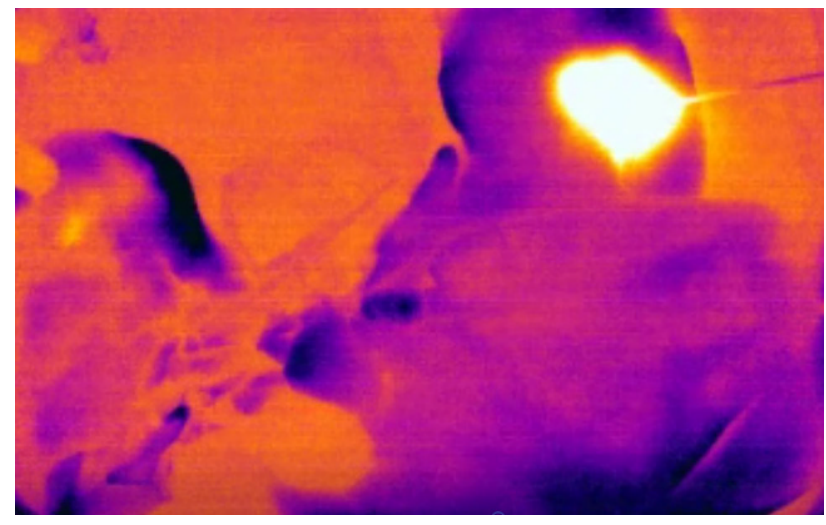

Fig. 1 Liver arts - the art of modern liver surgery. This picture is showing a perfused liver model, filmed in real time with a thermographic camera during an ablation procedure

vide a great overview incorporating a lot of their own research findings on liver regeneration and possible therapeutic treatment options to overcome liver dysfunction. Last but not least, the group of Brice Gayet, a pioneer in minimally invasive surgery, presents cur- rent technical advances and future perspectives in liver surgery (Ward et al.) (Fig. 1).

I really hope you find this special edition on contemporary liver surgery exciting and helpful for your daily work! Please do not hesitate to get in touch with any comments you would like to make; fruitful discussion is the beginning of any potential new development.

Lastly, I would like to thank all authors for their important and intricate contribution to this special edition in European Surgery.

Sincerely,

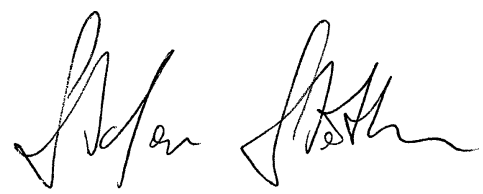

Stefan Stättner

Conflict of interest S. Stättner declares that he has no competing interests. 